\title{
Upper Extremity Rehabilitation of Stroke Patients
}

\author{
Jung-Ho Lee ${ }^{1}$, Dae-Hwan Lee ${ }^{2}$ \\ 1219-705 Dept. Physical Therapy, Kyungdong Univ., Bongpo-ri, Toseong-myeon, \\ Goseong-gun, Gangwon-do, Republic of Korea. \\ ${ }^{2}$ (Corresponding Author) 38430, Dept. Development, Y. H. Life Co., Ltd., B107, CU \\ technocenter, 13-13, Hayang-ro, Hayang-eup, Gyeongsan-si, Gyeongsangbuk-do, \\ Republic of Korea. \\ ${ }^{1}$ ljhcivapt@naver.com, ${ }^{2}$ dhlee8510@naver.com
}

\begin{abstract}
The object of this study was to investigate the influences of upper extremity exercises utilizing the upper extremity pattern of PNF, on the improvement of upper extremity functions in stroke patients. Ten subjects who agreed to participate in this study and satisfied the inclusion conditions were selected. They were randomized into the experimental and control groups. The experimental group $(n=5)$ received the upper extremity pattern of PNF after general physical therapy, whereas the control group $(n=5)$ received functional electrical stimulation after general physical therapy. To verify the intervention effects, the motor abilities before and after treatment were evaluated using the upper extremity distal part score of the Fugl-Meyer assessment (FMA) and the action research arm test. In addition, the exercise activity measurement chart was used to evaluate the activities of daily living. The FMA wrist and FMA hand, and ARAT score showed statistically significant differences between before and after the experiment in the experimental and control groups, respectively. In the comparison of treatment effects of the therapeutic intervention, the experimental group showed a statistically significant larger change compared to the control group.
\end{abstract}

Keywords: Stroke, Rehabilitation, Hand, Function, Nerve Regeneration

\section{Introduction}

The two major causes of stroke are cerebral infarction that blocks blood flow and cerebral hemorrhage that ruptures a cerebral blood vessel. Cerebral infarction occurs when a thrombus or embolus removed from a blood vessel because of blood clots that appear in the heart or other body parts blocks the cerebral blood vessel [1].

Cerebral infarction resulting from a blocked cerebral blood vessel can be divided into thrombosis and embolism, depending on the cause. Thrombosis refers to the formation of a thrombus in arteries that interferes with the blood flow in blood vessels, whereas embolism refers to the formation of a thrombus that flows along blood vessels and blocks a blood vessel in the brain, thus obstructing the blood flow [2].

Cerebral hemorrhage is a hemorrhagic stroke in which a weak blood vessel wall bursts and blood flows into the brain tissues. It is divided into intracerebral hemorrhage and subarachnoid hemorrhage. Intracerebral hemorrhage is a rupture of the cerebral blood vessel that causes

Article history:

Received (April 29, 2019), Review Result (May 26, 2019), Accepted (June 22, 2019) 
blood to accumulate in the brain tissue, which compresses the nerve and generates neurological symptoms. Subarachnoid hemorrhage is a hemorrhage in the space under the meninges where there is cerebrospinal fluid, which causes a high death rate and serious sequelae [3].

The purpose of this study was to investigate the effects of upper extremity exercises utilizing the upper extremity pattern of PNF, on the improvement of upper extremity functions in stroke patients.

\section{Subjects and Methods}

\subsection{Subjects}

Ten subjects who agreed to participate in this study and satisfied the inclusion conditions were selected. They were randomized into the experimental $(n=5)$ and control groups $(n=5)$.

The experimental group $(n=5)$ received the upper extremity pattern of PNF after general physical therapy, whereas the control group $(n=5)$ received functional electrical stimulation (FES) after general physical therapy Figure 1.

All the subjects comprehend the purpose of this study and provided written informed consent before participation according to the ethical standards of the Declaration of Helsinki.

\subsection{Research Methods}

To verify the intervention effects, the motor abilities before and after treatment were evaluated using the upper hand score of the Fugl-Meyer Assessment (FMA) and the Action Research Arm Test (ARAT). In addition, the exercise activity measurement chart was used to evaluate the activities of daily living.

In all assessments, a prior test was performed before the therapeutic intervention and a posttest was performed after the last therapeutic intervention.

For the upper extremity extension/flexion/internal rotation pattern among the upper extremity patterns of PNF, the subjects were asked to make a hooklying position in which they bent the hip and knee joints in a lying position, with the affected arm at eleven O'clock and the other arm comfortably placed beside the trunk.

For FES in this study, the FES treatment machine (Microstim, Germany) was used. FES was performed in four channels in a stable sitting position, with triceps, anterior fascia of the deltoid, wrist and finger extensors, and wrist and finger flexors.

For the statistical analysis in this study, the mean \pm standard deviation was described through descriptive statistics, using SPSS (v. 18.0; SPSS Inc., Chicago, IL). An independent sample ttest was performed to examine the intergroup differences, and a paired-sample t-test was performed for intragroup comparison between before and after data. The significance level of all data was $\mathrm{p}<0.05$.

\section{Results}

The FMA wrist and FMA hand, and ARAT score showed statistically significant differences between before and after the experiment in the experimental and control groups, respectively $(\mathrm{p}<0.05)$ Figure 2,3. In the comparison of treatment effects of the therapeutic intervention, the experimental group showed a statistically significant larger change compared to the control group $(\mathrm{p}<0.05)$ Figure 4. 


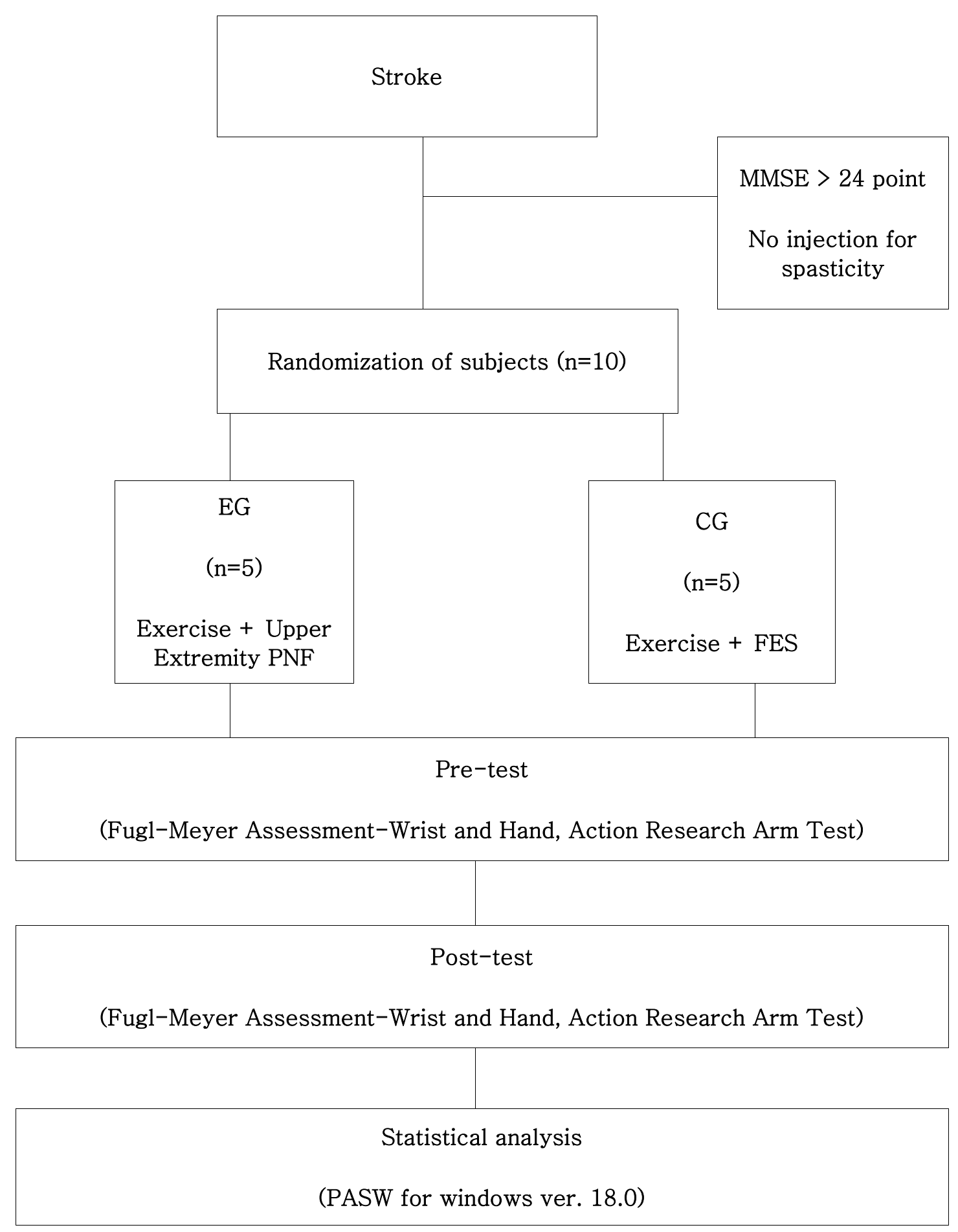

Figure 1. Flow chart 


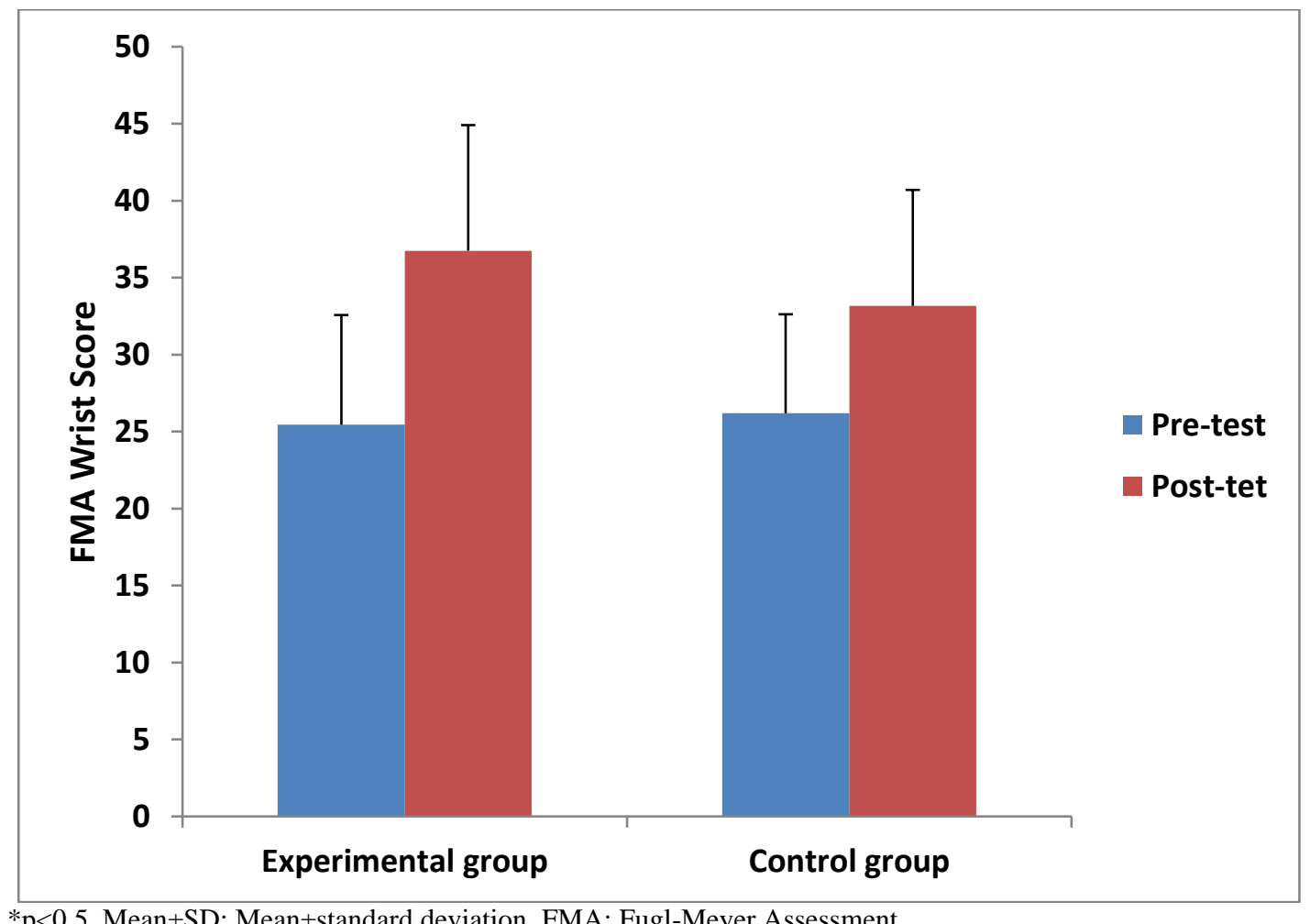

${ }^{*} \mathrm{p}<0.5$, Mean \pm SD: Mean \pm standard deviation, FMA: Fugl-Meyer Assessment

Figure 2. Comparison of FMA wrist score within groups

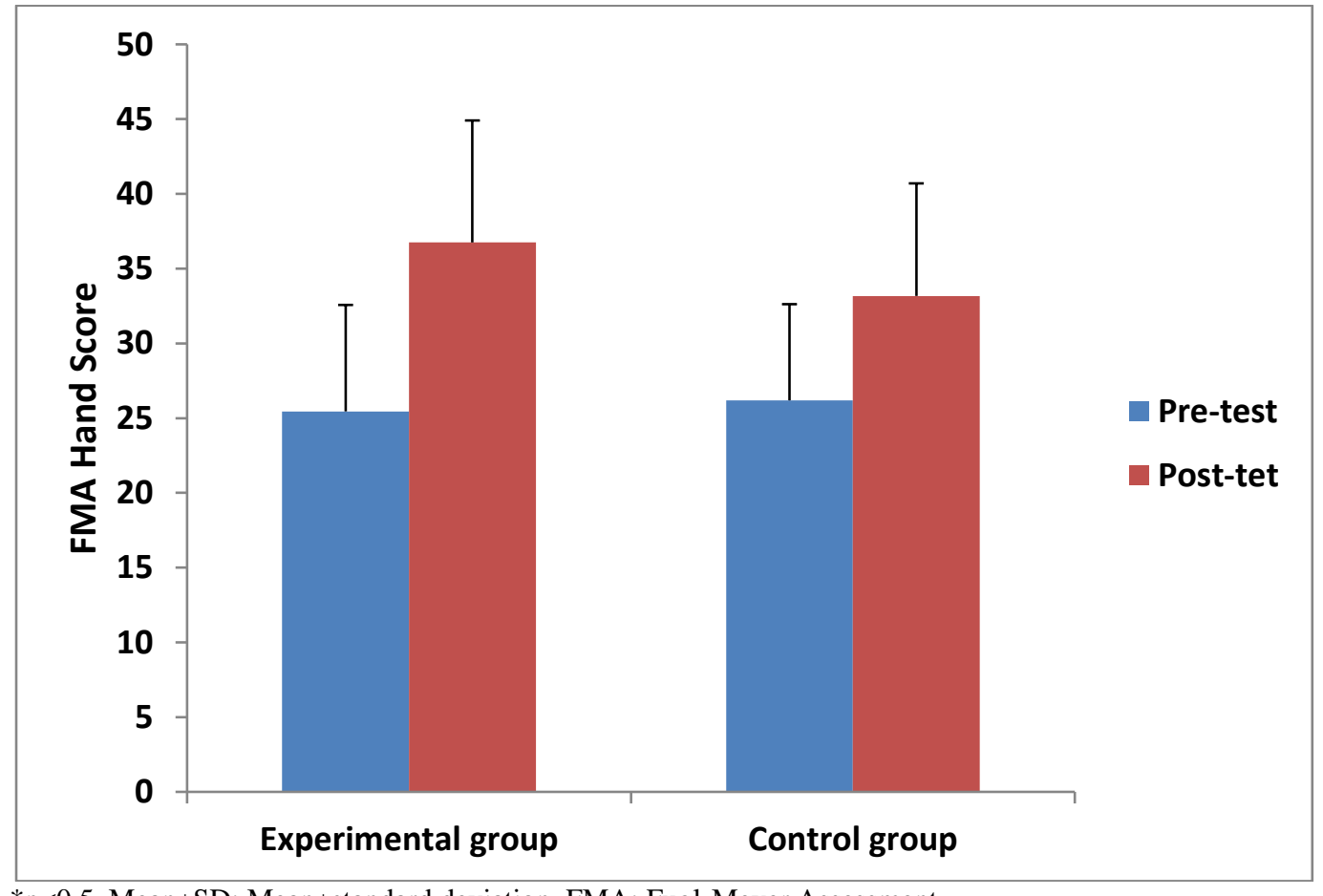

${ }^{*} \mathrm{p}<0.5$, Mean \pm SD: Mean \pm standard deviation, FMA: Fugl-Meyer Assessment

Figure 3. Comparison of FMA hand score within groups 


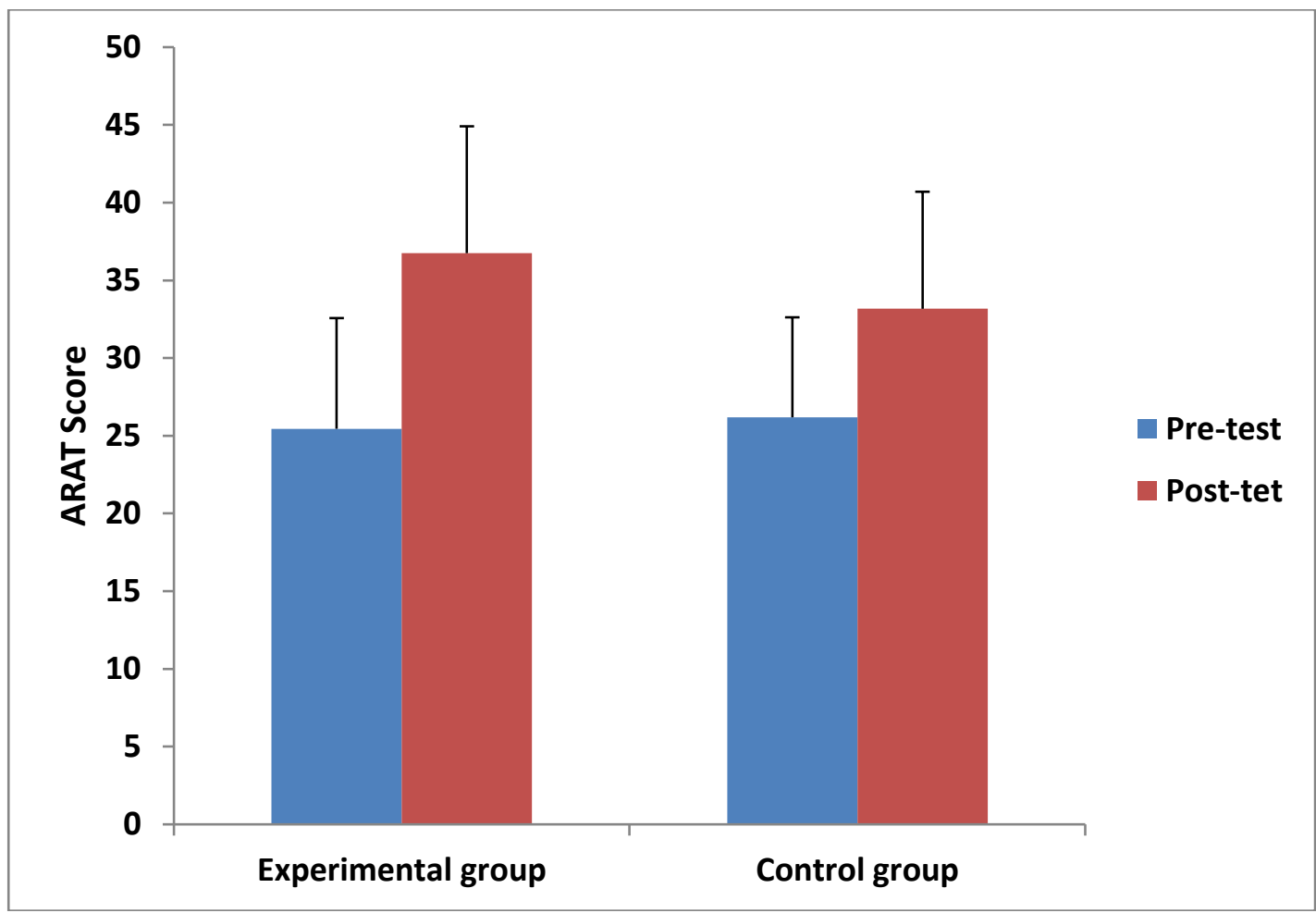

${ }^{*} \mathrm{p}<0.5$, Mean \pm SD: Mean \pm standard deviation, ARAT: Action Research Arm Test

Figure 4. Comparison of ARAT score within groups

\section{Discussion}

The two major causes of stroke are cerebral infarction that blocks blood flow and cerebral hemorrhage that ruptures a cerebral blood vessel. Cerebral infarction occurs when a thrombus or embolus removed from a blood vessel because of blood clots that appear in the heart or other body parts blocks the cerebral blood vessel [4].

Nervous system tend to have a very slow therapeutic recovery after damage and often show an incomplete degree of recovery. Nerve injury reduces the ability to control partial or overall motor nerve control in the area of the hernia and reduces the functional activities and quality of life caused by sensory and motor abilities disabilities [5].

The PNF therapy for the rehabilitation of stroke patients improves functions by stimulating the proprioceptive receptors in muscles and tendons and has been used as a representative therapeutic intervention for rehabilitation to improve the motor skills of patients with defection motions [6].

Various therapeutic interventions have been derived and developed in the category of PNF, including the promotion of postural reflexes, the use of gravity to promote the motion of weak muscles, the sue of eccentric contraction to promote the activity of agonist muscles, and the use of diagonal exercise pattern to promote the biarticular activity [7][8].

\section{Acknowledgements}

This work was supported by the National Research Foundation of Korea (NRF) grant funded by the Korea government (MSIT) (No-2019R1F1A1057731). 


\section{References}

[1] T. H. Wu, J. J. Lun, W. S. Chen, and F. C. Chong. "The electrophysiological and functional effect of shock wave on peripheral nerves”. Engineering in Medicine and Biology Society, pp.2369-2372. (2007) [DOI: 10.1109/IEMBS.2007.4352803]

[2] H. R. Rostami, M. Akbarfahimi, A. M. Hassani, A. R. Akbarinia, and S. Samani. "Occupation-based intervention versus rote exercise in modified constraint-induced movement therapy for patients with median and ulnar nerve injuries: A randomized controlled trial”. Clinical rehabilitation. Vol.31, pp.1087-1097. (2017) [DOI: 10.1177/0269215516672276]

[3] A. Santamato, A. Notarnicola, F. Panza, M. Ranieri, M. F. Micello, P. Manganotti, B. Moretti, F. Fortunato, S. Filoni, and P. Fiore, "Extracorporeal shock wave therapy versus electrical stimulation after botulinum toxin type a injection for post-stroke spasticity-a prospective randomized trial”, Ultrasound Med Biol, vol. 39, (2013), pp. 283-291.

[4] F. Mori, G. Koch, C. Foti, G. Bernardi, and D, Centonze, "The use of repetitive transcranial magnetic stimulation (rTMS) for the treatment of spasticity”, Prog Brain Res, vol. 175, (2009), pp. 429-439. [DOI: 10.1016/S0079-6123(09)17528-3]

[5] T. G. Kim, S. H. Bae, G. Y. Kim, and K. Y. Kim, "The effects of extracorporeal shock wave therapy on stroke patients with plantar fasciitis”, J Phys Ther Sci, vol. 27, (2015), pp. 523-526. [DOI: 10.1589/jpts.27.523]

[6] S. Mense, and U. Hoheisel, "Shock wave treatment improves nerve regeneration in the rat", Muscle Nerve, vol. 47, (2013), pp. 702-710. [DOI: 10.1002/mus.23631]

[7] J. H. Lee, Y. D. Choi, and C. W. Nam, "Extracorporeal Shock Wave Therapy Decreased Spasticity in Stroke", International Journal of IT-based Public Health Management, Vol. 4, (2017), pp. 43-48.

[8] A. I.Qureshi, A. D. Mendelow, and D. F. Hanley, "Intracerebral haemorrhage", The Lancet, Vol. 373, No.9675, (2009), pp. 1632-1644. [DOI: 10.1016/S0140-6736(09)60371-8] 